\title{
Image Retrieval Using Fuzzy Relevance Feedback and Validation with MPEG-7 Content Descriptors
}

\author{
M. Banerjee* and M.K. Kundu \\ Machine Intelligence Unit, Center for Soft Computing Research \\ Indian Statistical Institute \\ 203, B. T. Road, Kolkata 700 108, India \\ \{minakshi_r, malay\}@isical.ac.in
}

\begin{abstract}
Content-Based Image retrieval has emerged as one of the most active research directions in the past few years. In CBIR, selection of desired images from a collection is made by measuring similarities between the extracted features. It is hard to determine the suitable weighting factors of various features for optimal retrieval when multiple features are used. In this paper, we propose a relevance feedback frame work, which evaluates the features, from fuzzy entropy based feature evaluation index (FEI) for optimal retrieval by considering both the relevant as well as irrelevant set of the retrieved images marked by the users. The results obtained using our algorithm have been compared with the agreed upon standards for visual content descriptors of MPEG-7 core experiments.
\end{abstract}

Keywords: Content-Based image retrieval, fuzzy feature evaluation index, invariant moments, MPEG-7 feature descriptors.

\section{Introduction}

Digital images are widely used in many application fields such as biomedicine, education, commerce crime prevention, World Wide Web searching etc. This necessitates finding of relevant images from a database, by measuring similarities between the visual contents (color, texture, shape etc.) of the query images and those stored in the database and commonly known as Content-based image retrieval (CBIR) 1], 2, [3], 4].

Due to the vast activities in CBIR research over the last few years, Moving Pictures Expert Group (MPEG) has started the standardization activity for " Multimedia Content description Interface" in MPEG-7 [5], to provide standardization of feature descriptions for audiovisual data.

In a conventional CBIR, an image is usually represented by a set of features, where the feature vector is a point in the multidimensional feature space. However, images with high feature similarities may differ in terms of semantics. The

* M. Banerjee is grateful to the Department of Science and Technology, New Delhi, India, for supporting the work under grant (SR/WOS-A/ET-111/2003). 
discrepancy between the low level features like color, texture shape etc. and the high-level semantic concepts (such as sunset, flowers, outdoor scene etc.) is known as "semantic gap". To bridge this gap, users feedback may be used in an interactive manner which is popularly known as "relevance feedback" 6], 7], [8], 9]. The user rates the relevance of the retrieved images, from which the system dynamically learns the user's judgment to gradually generate better results. Owing to these facts, derivation and selection of optimal set of features, which can effectively model human perception subjectivity via relevance feedback, still remain a challenging issue.

Majority of the relevance feedback methods employ two approaches [10] namely, query vector moving technique and feature re-weighting technique to improve retrieval results. In the first approach, the query is reformulated by moving the vector towards positive / relevant examples and away from the negative examples, assuming that all positive examples will cluster in the feature space. Feature re-weighting method is used to enhance the importance of those components of a feature vector, that help in retrieving relevant images, while reducing the importance of the features that does not help. However in such cases, the selection of positive and negative examples, from a small number of samples having large number of features, still remains as a problem.

Relevance feedback techniques in CBIR, have mostly utilized information of the relevant images but have not made use of the information from irrelevant images. Zin et al., 11 have proposed a feature re-weighting technique by using both the relevant and the irrelevant information, to obtain more effective results. Recently, relevance feedback is considered as a learning and classification process, using classifiers like Bayesian classifiers [12, neural network [13]. However trained classifiers become less effective when the training samples are insufficient in number. To overcome such problems, active learning methods have been used in [14].

A fuzzy entropy based feature evaluation mechanism is provided for relevance feedback, combining information from both relevant and irrelevant images. The effectiveness of the proposed method is compared, with the results of Schema(XM) which uses the feature descriptors of MPEG-7 core experiments. The remaining sections are organized as follows : The section 2 describes the mathematical formulations for relevance feedback frame work. The experimental results and conclusion are described in section 3

\section{Estimation of Relative Importance of Different Features from Relevance Feedback}

Image retrieval using relevance feedback can be considered as a pattern classification problem. An effective relevance feedback system should be able to accumulate knowledge from small set of feedback images to form an optimal query. Each type of visual feature tends to capture only one aspect of the image property. To evaluate the importance of individual components for a particular query $I_{q r}$, the information from relevant and irrelevant images may be combined as follows : 
Let an image database $S_{d}$ be composed of $d$ distinct images, $I=\left\{I_{1}, I_{2}, \ldots, I_{d}\right\}$ where $I \in S_{d}$. The image $I$ is represented by a set of features $F=\left\{f_{q}\right\}_{q=1}^{N}$, where $f_{q}$ is the qth feature component in the $N$ dimensional feature space. The commonly used similarity function between the query image $I_{q r}$ and other images $I$, is represented as,

$$
D_{i s}\left(I, I_{q r}\right)=\sum_{q=1}^{N} w_{q}\left\|f_{q}(I)-f_{q}\left(I_{q r}\right)\right\|
$$

where $\left\|f_{q}(I)-f_{q}\left(I_{q r}\right)\right\|$ is the Euclidean distance between the qth component and $w_{q}$ is the weight assigned to the $q$ th feature component. The weights should be adjusted such that, the features have small variations over the relevant images and large variation over the irrelevant images. Let $k$ similar images $I_{s}=\left\{I_{1}, I_{2}, \ldots, I_{k}\right\}$ where, $I_{k} \in I_{s}$, are returned to the user. The information from relevant(intraclass) images $I_{r}$ and irrelevant (interclass) images $I_{i r}$ are combined to compute fuzzy feature evaluation index (FEI) proposed by Pal et al., [15, [16] in pattern classification problems.

Feature evaluation index: The fuzzy measure (FEI) is defined from interclass and intraclass ambiguities and explained as follows. Let $C_{1}, C_{2}, \ldots C_{j} \ldots C_{m}$ be the $\mathrm{m}$ pattern classes in an $\mathrm{N}$ dimensional $\left(f_{1}, f_{2}, f_{q}, \ldots f_{N}\right)$ feature space where class $C_{j}$ contains, $n_{j}$ number of samples. It is shown that fuzzy entropy $\left(H_{q j}\right)$ 17. gives a measure of 'intraset ambiguity' along the qth co-ordinate axis in $C_{j}$. The entropy of a fuzzy set, having $n_{j}$ points in $C_{j}$ is computed as,

$$
\left.H(A)=\left(\frac{1}{n_{j} \ln 2}\right) \sum_{i} S_{n}\left(\mu_{(} f_{i q j}\right)\right) ; i=1,2 \ldots n_{j}
$$

where the Shannon's function,$\left(S_{n} \mu\left(f_{i q j}\right)\right)=-\mu\left(f_{i q j}\right) \ln \mu\left(f_{i q j}\right)-\left\{1-\mu\left(f_{i q j}\right)\right\} \ln$ $\left\{1-\mu\left(f_{i q j}\right)\right\}$

For computing $\mathrm{H}$ of $C_{j}$ along qth component, a standard S-type function shown in Fig. 1 is considered. At $b$ (cross over point), $\mathrm{S}(\mathrm{b} ; \mathrm{a}, \mathrm{b}, \mathrm{c})=0.5$. Similarly at $c$ (shoulder point) $S(c ; a, b, c)=1.0$ and at $a$ (feet point) $\mathrm{S}(\mathrm{a} ; \mathrm{a}, \mathrm{b}, \mathrm{c})=0.0$.

The parameters are set as follows. $b=\left(f_{q j}\right) a v, c=b+\max \left\{\mid\left(f_{q j}\right) a v-\right.$ $\left.\left(f_{q j}\right) \max ||,\left(f_{q j}\right) a v-\left(f_{q j}\right) \min \mid\right\}, a=2 b-c$ where $\left(f_{q j}\right) a v,\left(f_{q j}\right) \max ,\left(f_{q j}\right) \min$ denote the mean, maximum and minimum values respectively computed along the qth co-ordinate axis over all the $n_{j}$ samples in $c_{j}$.

The values of $H$ are 1.0 at $b=\left(f_{q j}\right) a v$ and would tend to zero when moved away from $b$ towards either $c$ or $a$ of the S function, where $\mu(b)=\mu\left(f_{q j}\right) a v=0.5$, eqn. (2). Selecting $b=\left(f_{q j}\right) a v$ indicates that, the cross over point is near to the query feature component. Higher value of $H$, indicates more samples having $\mu(f)$ equal to 0.5. i.e., cluster around the mean value, resulting in less internal scatter within the class. After combining the classes $C_{j}$ and $C_{k}$ the mean, maximum and minimum values $\left(f_{q k j}\right)$ av, $\left(f_{q j k}\right) \max ,\left(f_{q j k}\right)$ min respectively of qth dimension over the samples $\left(n_{j}+n_{k}\right)$ are computed similarly to evaluate "interset ambiguity" $H_{q j k}$, where $n_{k}$ are the samples in class $C_{k}$. 
The criteria of a good feature is that, it should be nearly invariant within class, while having differences between patterns of different classes 15. The value of $\mathrm{H}$ would therefore decrease, after combining $C_{j}$ and $C_{k}$ as the goodness of the qth feature in discriminating pattern classes $C_{j}$ and $C_{k}$ increases. Considering the two types of ambiguities, the proposed Feature evaluation index (FEI) for the qth feature is,

$$
\left(F E I_{q}\right)=\frac{H_{q j k}}{H_{q j}+H_{q k}}
$$

Lower the value of $F E I_{q}$, higher is the quality of importance of the qth feature in recognizing and discriminating different classes. The user marks the relevant and irrelevant set from 20 returned images. To evaluate the importance of the qth feature, the qth component of the retrieved images is considered. i.e., $I^{(q)}$ $=\left\{I_{1}^{(q)}, I_{2}^{(q)}, I_{3}^{(q)}, \ldots . I_{k}^{(q)}\right\}$. Value of $H_{q j}$ is computed from $I_{r}^{(q)}=\left\{I_{r 1}^{(q)}, I_{r 2}^{(q)}, I_{r 3}^{(q)}\right.$, $\left.\ldots I_{r k}^{(q)}\right\}$. Similarly $H_{q k}$ is computed from the set of images, $I_{i r}^{(q)}=\left\{I_{i r 1}^{(q)}, I_{i r 2}^{(q)}, I_{i r 3}^{(q)}\right.$, $\left.\ldots . . I_{i r k}^{(q)}\right\} . H_{q k j}$ is computed combining both the sets.

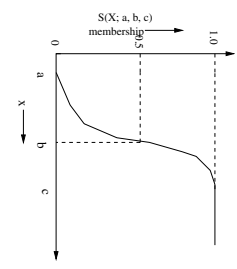

Fig. 1. S-type membership function

Effect of sample size on evaluating importance: To address the issue like, how to manage with limited number of returned images, we study the effect of image sample size on the FEI values. We combine two distinct categories of images. We mark category (1) as relevant and those from category (2) as irrelevant. We increase the sample size of the relevant and irrelevant images (double, triple, half, etc). We also test with other combinations. The FEI values computed from different combinations are shown in Table.1. As seen from the table, the order of the values obtained for $(F E I)_{q}$ is same for a particular query category.

Adjustment of weights: The marking of relevant and irrelevant images is subjective. One may emphasize more on similarity between one feature (eg., color) than some other features ( eg., shape), when comparing two images. Accordingly the weights need to be adjusted. For the query feature vector $F$. The individual components of relevant images are expected to vary within a smaller range say $(\epsilon)$ and may be represented as .

$$
I_{r}=\left\{I_{j} \in I_{s}: \frac{\delta f_{q}}{|F|} \leq \epsilon\right\}
$$


In the first pass, all features are considered to be equally important. Hence $w_{1}=w_{2}, \ldots=w_{q}=1$. The feature spaces of the relevant images are therefore altered in a similar fashion after updating the components with $w_{q}$. As a result, the ranks of the relevant images are not affected much. For irrelevant images, one feature component may be very close to the query, whereas other feature component may be far away from the query feature. But the magnitude of the similarity vector may be close to the relevant ones. These images may be characterized as,

$$
I_{i r}=\left\{I_{j} \in I_{s}: \frac{\delta f_{q 1}}{|F|} \gg \epsilon \text { and } \frac{\delta f_{q 2}}{|F|} \ll \epsilon\right\}
$$

After the features of the query and the stored images $\left(S_{d}\right)$ have been updated with the FEI values, the weighted components are expected to dominate over feature space such that, the rank of the irrelevant ones are pulled down. We have tested the results, from updating the weights with $w_{q}=F E I_{q}{ }^{2},{\frac{1}{F E I_{q}}}^{2}$ and obtained better results from $w_{q}=F E I_{q}{ }^{2}$, in majority of the cases. Intuitively this depends on how the combination of important features dominate over the others for a particular query. Importance of a features is decided from the decreasing order of (FEI) values. In order to further enhance the effect of the important features, we introduce another multiplying factor $t_{q f}$ to make the component more dominating The weights of the individual features for successive iterations are expressed as follows ; $w_{q f}=t_{q f} \times\left(F E I_{q f}\right)^{2}$ where the value of $t_{q f}$ are chosen as $\{1.0,0.1\}$. to get better results.

Features used for characterizing an image: The features are computed from a set of moments invariant to rotation, translation and scaling. The moments $m_{p q}$ of order $\mathrm{p}$ and $\mathrm{q}$ of a function $f(x, y)$ for discrete images, are usually approximated as,

$$
m_{p q}=\sum_{x} \sum_{y} x^{p} y^{q} f(x, y)
$$

The centralized moments are expressed as, $\mu_{p q}=\sum_{x} \sum_{y}(x-\bar{x})(y-\bar{y}) f(x, y)$ where $\bar{x}=\frac{m_{10}}{m_{00}}, \bar{y}=\frac{m_{01}}{m_{00}}$

The normalized central moments are computed as, $\eta_{p q}=\frac{\mu_{p q}}{\mu_{00}^{\gamma}}$ where $\gamma=\frac{p+q}{2}+1$ for $p+q=2,3, \ldots$. A set of seven moments invariant to translation, rotation and scale can be computed from $\eta_{p q}$, from which $\theta=\eta_{02}+\eta_{02}$ is considered. Let $I(x, y)_{R}, I(x, y)_{G}, I(x, y)_{B}$ represent the R,G,B component planes of the image matrix $I(x, y)$ and $I_{s}(x, y)_{R} I_{s}(x, y)_{G} I_{s}(x, y)_{B}$ represnt the component planes of the representative locations 18 shown in Fig,2. Six values of invariant moments $(\theta)$ are computed from the described component planes, to represent the feature vector. 


\section{Experimentation}

The performance of image retrieval system is tested upon SIMPLIcity images which consists of 1000 images from 10 different categories. The average precision value from each category (randomly chosen queries), after retrieving $(10,20,40)$ images are shown in Table 2. Altough the performance mostly depends uopn the choice of features, it is observed that, the results almost converged after two iterations. A retrieval result is shown in Fig 3 .

Complexity. The time complexity $\mathrm{T}(\mathrm{n})$ for matching and sorting our results is represented as, $\mathrm{T}(\mathrm{n})=\mathrm{O}(\mathrm{ND})+\mathrm{O}(\mathrm{NlogN})$. The complexity involved in computing Euclidean distance is $\mathrm{O}(\mathrm{ND})$ where $D$ is the number of components within the feature vector. Sorting of $(\mathrm{N})$ images with quick sort of $\mathrm{O}(\mathrm{Nlog} N)$. As $(\mathrm{D}=6)$. Hence $D \ll \log N$. Therefore $T(n) \simeq O(N \log N)$.

Table 1. Feature evaluation index

\begin{tabular}{|c|c|c|c|c|c|c|}
\hline Images(Intra, Inter $)$ & $\left(F E I_{1}\right)^{2}$ & $\left(F E I_{2}\right)^{2}$ & $\left(F E I_{3}\right)^{2}$ & $\left(F E I_{4}\right)^{2}$ & $\left(F E I_{5}\right)^{2}$ & $\left(F E I_{6}\right)^{2}$ \\
\hline$(10,10)$ & 0.207 & 0.115 & 0.199 & 0.351 & 0.351 & 0.351 \\
\hline$(20,20)$ & 0.207 & 0.115 & 0.199 & 0.351 & 0.351 & 0.351 \\
\hline$(30,30)$ & 0.220 & 0.121 & 0.195 & 0.350 & 0.350 & 0.350 \\
\hline$(5,5)$ & 0.309 & 0.151 & 0.247 & 0.349 & 0.349 & 0.349 \\
\hline$(5,10)$ & 0.289 & 0.164 & 0.265 & 0.306 & 0.306 & 0.306 \\
\hline$(10,5)$ & 0.433 & 0.213 & 0.401 & 0.534 & 0.534 & 0.534 \\
\hline
\end{tabular}

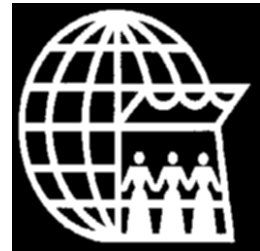

(a)

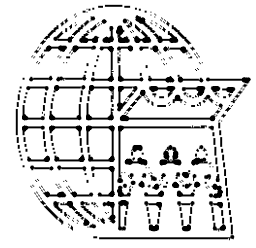

(b)

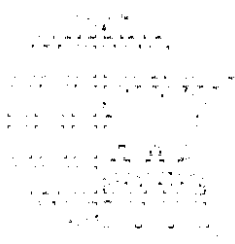

(c)

Fig. 2. (a) Original image (b) Edge signature on which the high curvature region marked as $\left(^{*}\right)(\mathrm{c})$ corner signature

Comparison with MPEG-7: MPEG-7 [19] is an ISO/IEC standard, which provides a collection of specific, agreed upon standard (audio, visual) descriptors. MPEG-7 experimentation Model (XM) software [5] is the frame work of all the reference codes, and make use of MPEG-7 visual descriptors. These can serve as a test bed for evaluation and comparison of features in CBIR context. The features of XM commonly used as standard visual content descriptors for still images are listed in Table. 3. 

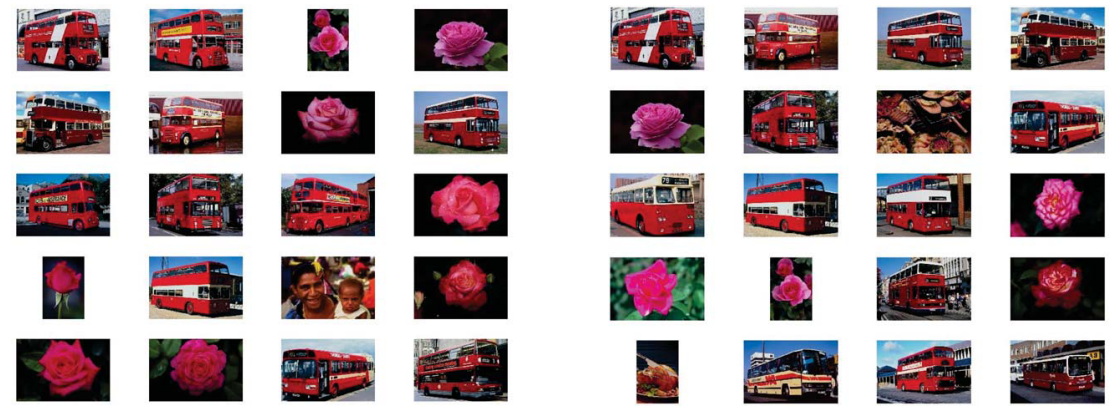

(a)

(b)

Fig. 3. Retrieved results using set(A) (a) first set of candidates (b) After feature evaluation with $(\mathrm{FEI})$, (iteration 1$), t_{i}{ }^{*} F E I_{i=1}^{N}=\{1 \times 0.22,1 \times 0.32,1 \times 0.39,0.1 \times 0.36$, $0.1 \times 0.32,0.1 \times 0.37\}$. The top left image is the query image.

Table 2. Average precision $\%$ from our algorithm

\begin{tabular}{|c|c|c|c|c|c|c|}
\hline \multirow{2}{*}{ Category } & \multicolumn{3}{|c|}{ unweighted features } & \multicolumn{3}{c|}{ weighted features (iteration 1) } \\
\cline { 2 - 7 } & 10 images & 20 img. & 40 img. & 10 img. & 20 img. & 40 img. \\
\hline Africa & 68.20 & 60.25 & 55.03 & 70.50 & 61.00 & 58.00 \\
\hline Beach & 70.00 & 55.23 & 50.60 & 71.48 & 56.23 & 52.58 \\
\hline Building & 70.26 & 60.50 & 54.46 & 72.40 & 63.67 & 56.00 \\
\hline Bus & 80.00 & 70.59 & 60.67 & 81.45 & 72.77 & 62.67 \\
\hline Dinosaur & 100.0 & 95.0 & 90.7 & 100.0 & 95.0 & 92.0 \\
\hline Elephant & 83.5 & 75.5 & 65.8 & 85.0 & 77.0 & 66.0 \\
\hline Flower & 90.0 & 80.5 & 70.6 & 92.0 & 83.0 & 71.2 \\
\hline Horses & 100.0 & 90.0 & 80.5 & 100.0 & 95.0 & 83.0 \\
\hline Mountains & 70.5 & 65.8 & 60.9 & 72.0 & 68.0 & 62.0 \\
\hline Food & 60.8 & 55.8 & 53.40 & 62.0 & 57.0 & 55.20 \\
\hline
\end{tabular}

These features have been rigorously tested in the standardization process. We have seen the query results for all the classes available in Schema ( Beaches, buildings, horses, cars, flowers etc. )which utilize the MPEG-7 visual descriptors. The performance is compared for the case of evaluating overall similarity between images from precision rate defined as, $P_{r}=\left(1 / n_{1}\right) \sum r / n$ where, the system retrieves $r$ images that belongs to the same class $C 1$ from $n$ retrieved images. $n_{1}$ is the number images queried from category $C 1$. The images from other categories are taken as outliers. The results are shown in Figs. 4 and 5 . Our results can be fairly compared with MPEG-7 visual descriptors, as seen from Figs. 4 and 5. Although the initial precision is found better for SchemaXM as shown in Fig. 4(a) and Fig 5 (b) but our algorithm fairly catches the results. 
Table 3. Standard Visual content descriptors of MPEG-7

\begin{tabular}{|c|c|c|}
\hline Color Descriptors & Texture Descriptors & Shape Descriptors based \\
\hline \hline Dominant Colors & Edge Histogram & Region Based Shape \\
\hline Scalable Color & Homogeneous Texture & Contour Based Shape \\
\hline Color Layout & & \\
\hline Color Structure & & \\
\hline
\end{tabular}

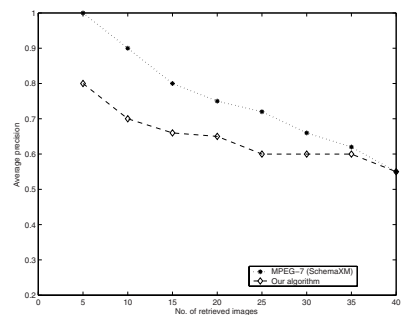

(a)

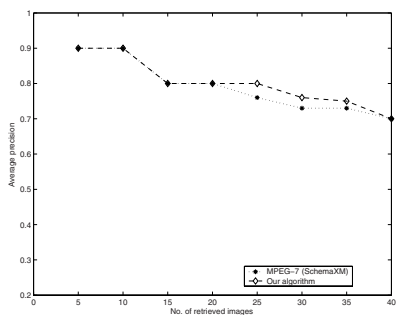

(b)

Fig. 4. Comparison with MPEG-7 visual descriptors (a) Category beaches (b) Flowers

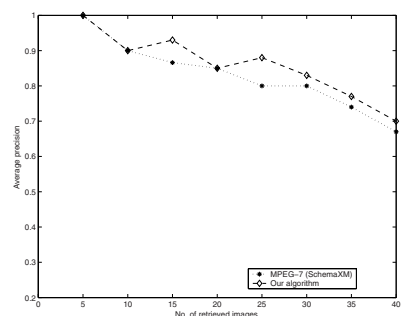

(a)

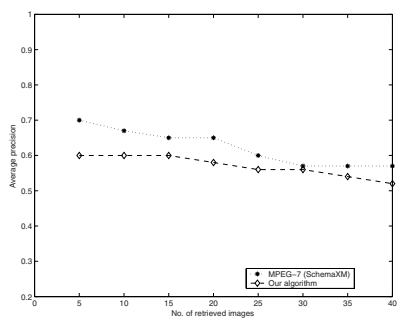

(b)

Fig. 5. Comparison with MPEG-7 visual descriptors (a) Category horse (b) Vehicles

Conclusion: In the current work, a fuzzy entropy based relevance feedback, framework for image retrieval is proposed. We intend to test the effectiveness of the system, using simple features, invariant moments in the present case. We plan to improve our scheme by associating text and other features, for better compatibility with MPEG-7 and better semantic modeling.

\section{References}

1. Smeulders, A.W.M., Worring, M., Santini, S., Gupta, A., Jain, R.: Content-based image retrieval at the end of the early years. IEEE Transactions on Pattern Analysis and Machine Intelligence 22(12), 1349-1380 (2000)

2. Kunttu, I., Lepisto, L., Rauhamaa, J., Visa, A.: Multiscale fourier descriptors for defect image retrieval. Pattern Recognition Letters 27(2), 123-132 (2006) 
3. Gevers, T., Smeulders, A.W.M.: Combining color and shape invariant features for image retrieval. Image and Vision computing 17(7), 475-488 (1999)

4. Chen, Y., Wang, J.Z., Krovetz, R.: Clue: Cluster-based retrieval of images by unsupervised learning. IEEE Transactions on Image Processing 14(8), 1187-1201 (2005)

5. MPEG-7 Multimedia Description Schemes (XM). ISO/IEC JTC1/SC29/WG11 N3914 (2001)

6. Han, J., Ngan, K.N., Li, M., Zhang, H.J.: A memory learning framework for effective image retrieval. IEEE Transactions on Image Processing 14(4), 521-524 (2005)

7. Chang, F.C., Hang, H.M.: A relevance feedback image retrieval scheme using multiinstance and pseudo image concepts. IEICE Transactions on Information and Systems D(5), 1720-1731 (2006)

8. Yin, P.Y., Bhanu, B., Chang, K.C., Dong, A.: Integrating relevance feedback techniques for image retrieval using reinforcement learning. IEEE Transactions on Pattern Analysis and Machine Intelligence 27(10), 1536-1551 (2005)

9. Lim, J.H., Jin, J.S.: Combining intra-image and inter-class semantics for consumer image retrieval. Pattern Recognition 38(6), 847-864 (2005)

10. Rui, Y., Huang, T.S., Mehrotra, S.: Content-based image retrieval with relevance feedback in MARS. In: Proceedings of the IEEE International Conference on Image Processing, pp. 815-818 (1997)

11. Jin, Z., King, I., Li, X.Q.: Content-based retrieval by relevance feedback. In: Laurini, R. (ed.) VISUAL 2000. LNCS, vol. 1929, pp. 521-529. Springer, Heidelberg (2000)

12. ves, E.D., Domingo, J., Ayala, G., Zuccarello, P.: A novel bayesian framework for relevance feedback in image content-based retrieval systems. Pattern Recognition 39(9), 1622-1632 (2006)

13. Qian, F., Zhang, B., Lin, F.: Constructive learning algorithm-based rbf network for relevance feedback in image retrieval. In: Bakker, E.M., Lew, M.S., Huang, T.S., Sebe, N., Zhou, X.S. (eds.) CIVR 2003. LNCS, vol. 2728, pp. 352-361. Springer, Heidelberg (2003)

14. He, X., King, O., Ma, W., Li, M., Zhang, H.J.: Learning a semantic space from user's relevance feedback for image retrieval. IEEE transactions on Circuits and Systems for Video technology 2003 13(1) (2003)

15. Pal, S.K., Chakraborty, B.: Intraclass and interclass ambiguities (fuzziness) in feature evaluation. Pattern Recognition Letters 2, 275-279 (1984)

16. Pal, S.K., Chakraborty, B.: Fuzzy set theoretic measures for automatic feature evaluation. IEEE Transactions on Systems, Man and Cybernatics 16(5), 754-760 (1986)

17. Pal, S.K., Majumder, D.D.: Fuzzy mathematical Approach to Pattern Recognition. Willey Eastern Limited, New York (1985)

18. Banerjee, M., Kundu, M.K.: Content Based Image Retrieval with Multiresolution Salient points. In: ICVGIP 2004. Fourth Indian Conference Computer Vision, Graphics and Image Processing, India, pp. 399-404 (2004)

19. Manjunath, B.S., Salembier, P., S, T.: Introduction to MPEG-7: Multimedia Content description Interface. John Wiley and Sons, Inc, USA (2002) 